\title{
Circular Chosen-Ciphertext Security with Compact Ciphertexts
}

\author{
Dennis Hofheinz ${ }^{\star}$ \\ Karlsruhe Institute of Technology
}

\begin{abstract}
A key-dependent message (KDM) secure encryption scheme is secure even if an adversary obtains encryptions of messages that depend on the secret key. Such key-dependent encryptions naturally occur in scenarios such as harddisk encryption, formal cryptography, or in specific protocols. However, there are not many provably secure constructions of KDM-secure encryption schemes. Moreover, only one construction, due to Camenisch, Chandran, and Shoup (Eurocrypt 2009) is known to be secure against active (i.e., CCA) attacks.

In this work, we construct the first public-key encryption scheme that is KDM-secure against active adversaries and has compact ciphertexts. As usual, we allow only circular key dependencies, meaning that encryptions of arbitrary entire secret keys under arbitrary public keys are considered in a multi-user setting.

Technically, we follow the approach of Boneh. Halevi. Hamburg. and Ostrovskv (Crypto 2008) to KDM security, which however only achieves security against passive adversaries. We explain an inherent problem in adapting their techniques to active security, and resolve this problem using a new technical tool called "lossy algebraic filters" (LAFs). We stress that we significantly deviate from the approach of Camenisch, Chandran, and Shoup to obtain KDM security against active adversaries. This allows us to develop a scheme with compact ciphertexts that consist only of a constant number of group elements.
\end{abstract}

Keywords: key-dependent messages, chosen-ciphertext security, publickey encryption.

\section{Introduction}

KDM Security. An encryption scheme is key-dependent message (KDM) secure if it is secure even against an adversary who has access to encryptions of messages that depend on the secret key. Such a setting arises, e.g., in harddisk encryption [10], computational soundness results in formal methods [7, 2], or specific protocols [13]. KDM security does not follow from standard security [1, 15], and there are indications [19, 5] that KDM security (at least in its most

\footnotetext{
* Supported by DFG grant GZ HO 4534/2-1.
} 
general form) cannot be proven using standard techniques; it seems that dedicated constructions and proof techniques are necessary 1

The BHHO Approach to KDM-CPA Security. Boneh, Halevi, Hamburg, and Ostrovsky [10] (henceforth BHHO) were the first to construct and prove a public-key encryption (PKE) scheme that is KDM secure under chosen-plaintext attacks (KDM-CPA-secure) in the standard model, under the Decisional DiffieHellman (DDH) assumption. While they did not prove their scheme secure under messages that arbitrarily depend on the secret key, their result encompasses the important case of circular (CIRC-CPA) security. Loosely speaking, a PKE scheme is circular secure if it is secure even in a multi-user setting where encryptions of arbitrary secret keys under arbitrary public keys are known. This notion is sufficient for certain applications [13], and can often be extended to stronger forms of KDM security [5, 12]. Inspired by BHHO, KDM-CPA-secure PKE schemes from other computational assumptions followed [4, 11, 25].

Since we will be using a similar approach, we give a high-level intuition of BHHO's approach. The crucial property of their scheme is that it is publicly possible to construct encryptions of the secret key (under the corresponding public key). Thus, encryptions of the secret key itself do not harm the (INDCPA) security of that scheme. Suitable homomorphic properties of both keys and ciphertexts allow to extend this argument to circular security (for arbitrarily many users/keys), and to affine functions of all keys.

\section{Why the BHHO Approach Fails to Achieve KDM-CCA Security.} When considering an active adversary, we require a stronger form of KDM security. Namely, KDM-CCA, resp. CIRC-CCA security requires security against an adversary who has access to key-dependent encryptions and a decryption oracle. (Naturally, to avoid a trivial notion, the adversary is not allow to submit any of those given KDM encryptions to its decryption oracle.) Now if we want to extend BHHO's KDM-CPA approach to an adversary with a decryption oracle, the following problem arises: since it is publicly possible to construct (fresh) encryptions of the secret key, an adversary can generate such an encryption and then submit it to its decryption oracle, thus obtaining the full secret key. Hence, the very property that BHHO use to prove KDM-CPA security seemingly contradicts chosen-ciphertext security.

Our Technical Tool: Lossy Algebraic Filters (LAFs). Before we describe our approach to KDM-CCA security, let us present the core technical tool we use. Namely, a lossy algebraic filter (LAF) is a family of functions, indexed by a public key and a tag. A function from that family takes a vector $X=\left(X_{i}\right)_{i=1}^{\mathfrak{n}}$ as input. Now if the tag is lossy, then the output of the function reveals only a linear combination of the $X_{i}$. If the tag is injective, however, then so is the function. We require that there are many lossy tags, which however require a special trapdoor to be found. On the other hand, lossy and injective tags are computationally

${ }^{1}$ We mention, however, that there are semi-generic transformations that enhance the KDM security of an already "slightly" KDM-secure scheme [5, 12, 3]. 
indistinguishable. This concept is very similar to (parameterized) lossy trapdoor functions [27], and in particular to all-but-many lossy trapdoor functions (ABMLTFs [20]). However, we do not require efficient inversion, but we do require that lossy functions always reveal the same linear combination about the input. In particular, evaluating the same input under many lossy tags will still leave the input (partially) undetermined.

We give a construction of LAFs under the Decision Linear (DLIN) assumption in pairing-friendly groups. Similar to ABM-LTFs, lossy tags correspond to suitably blinded signatures. (This in particular allows to release many lossy tags, while still making the generation of a fresh lossy tag hard for an adversary.) However, unlike with ABM-LTFs, functions with lossy tags always release the same information about its input. Our construction has compact tags with $\mathbf{O}(1)$ group elements, which will be crucial for our KDM-CCA secure encryption scheme 2

Our Approach to KDM-CCA Security. We can now describe our solution to the KDM-CCA dilemma explained above. We will start from a hybrid between the BHHO-like PKE schemes of Brakerski and Goldwasser [11], resp. Malkin et al. 25]. This scheme has compact ciphertexts $(\mathbf{O}(1)$ group elements), and its KDM-CPA security can be proved under the Decisional Composite Residuosity (DCR) assumption. As with the BHHO scheme, the scheme's KDM-CPA security relies on the fact that encryptions of its secret key can be publicly generated. Essentially, our modification consists of adding a suitable authentication tag to each ciphertext. This authentication tag comprises the (encrypted) image of the plaintext message under an LAF. During decryption, a ciphertext is rejected in case of a wrong authentication tag.

In our security proof, all authentication tags for the key-dependent encryptions the adversary gets are made with respect to lossy filter tags. This means that information-theoretically, little information about the secret key is released (even with many key-dependent encryptions, resp. LAF evaluations). However, any decryption query the adversary makes must refer (by the LAF properties) to an injective tag. Hence, in order to place a valid key-dependent decryption query, the adversary would have to guess the whole (hidden) secret key 3

Thus, adding a suitable authentication tag allows us to leverage the techniques by $\mathrm{BHHO}$, resp. Brakerski and Goldwasser, Malkin et al. to chosen-ciphertext attacks. In particular, we obtain a CIRC-CCA-secure PKE scheme with compact ciphertexts (of $\mathbf{O}(1)$ group elements). We prove security under the conjunction of the following assumptions: the DCR assumption (in $\mathbb{Z}_{N^{3}}^{*}$ ), the DLIN assumption

\footnotetext{
${ }^{2}$ The size of the LAF public key depends on the employed signature scheme. In our main construction, we use Waters signatures, which results in very compact tags, but public keys of $\mathbf{O}(k)$ group elements, where $k$ is the security parameter. Alternatively, at the end of Section 3.1, we sketch an LAF with constant-size (but larger than in our main construction) tags and constant-size public keys.

${ }^{3}$ We will also have to protect against a re-use of (lossy) authentication tags, and "ordinary", key-independent chosen-ciphertext attacks. This will be achieved by a combination of one-time signatures and 2-universal hash proof systems [16, 24, 22].
} 
(in a pairing-friendly group), and the DDH assumption (somewhat curiously, in the subgroup of order $(P-1)(Q-1) / 4$ of $\mathbb{Z}_{N^{3}}^{*}$, where $\left.N=P Q\right) \square$

Relation to Camenisch et al.'s CIRC-CCA-secure Scheme. Camenisch, Chandran, and Shoup [14] present the only other known CIRC-CCA-secure PKE scheme in the standard model. They also build upon BHHO techniques, but instead use a Naor-Yung-style double encryption technique [26] to achieve chosenciphertext security. As an authentication tag, they attach to each ciphertext a non-interactive zero-knowledge proof that either the encryption is consistent (in the usual Naor-Yung sense), or that they know a signature for the ciphertext. Since they build on the original, DDH-based BHHO scheme, they can use Groth-Sahai proofs [18] to prove consistency. Compared to our scheme, their system is less efficient: they require $\mathbf{O}(k)$ group elements per ciphertext, and the secret key can only be encrypted bitwise. However, their sole computational assumption to prove circular security is the DDH (or, more generally, $k$-Linear) assumption in pairing-friendly groups. One thing to point out is their implicit use of a signature scheme. Their argument is conceptually not unlike our LAF argument. However, since they can apply a hybrid argument to substitute all key-dependent encryptions with random ciphertexts, they only require one-time signatures. Furthermore, the meaning of "consistent ciphertext" and "proof" in our case is very different. (Unlike Camenisch et al., we apply an argument that rests on the information that the adversary has about the secret key.)

Note about Concurrent Work. In a work concurrent to ours, Galindo, Herranz, and Villar [17] define and instantiate a strong notion of KDM security for identity-based encryption (IBE) schemes. Using the IBE $\rightarrow$ PKE transformation of Boneh, Canetti, Halevi, and Katz [9], they derive a KDM-CCAsecure PKE scheme. Their concrete construction is entropy-based and achieves only a bounded form of KDM security, much like the KDM-secure SKE scheme from [23]. Thus, while their ciphertexts are very compact, they can only tolerate a number of (arbitrary) KDM queries that is linear in the size of the secret key. In particular, it is not clear how to argue that the encryption of a full secret key in their scheme is secure.

\section{Preliminaries}

Notation. For $n \in \mathbb{N}$, let $[n]:=\{1, \ldots, n\}$. Throughout the paper, $k \in \mathbb{N}$ is the security parameter. For a finite set $\mathcal{S}, s \leftarrow \mathcal{S}$ denotes the process of sampling $s$ uniformly from $\mathcal{S}$. For a probabilistic algorithm $A, y \leftarrow A(x ; R)$ denotes the process of running $A$ on input $x$ and with randomness $R$, and assigning $y$ the

\footnotetext{
${ }^{4}$ Very roughly, we resort to the DDH assumption since we release partial information about our secret keys. Whereas the argument of [11, 25] relies on the fact that the secret key $s k$ is completely hidden modulo $N$, where computations take place in $\mathbb{Z}_{N}$, we cannot avoid to leak some information about $s k \bmod N$ by releasing LAF images of $s k$. However, using a suitable message encoding, we can argue that $s k$ is completely hidden modulo the coprime order $(P-1)(Q-1) / 4$ of quadratic residues modulo $N$, which enables a reduction to the DDH assumption.
} 
result. We write $y \leftarrow A(x)$ for $y \leftarrow A(x ; R)$ with uniformly chosen $R$. If $A$ 's running time is polynomial in $k, A$ is called probabilistic polynomial-time (PPT).

Standard Definitions. Due to lack of space, we postpone some standard definitions to the full version 21]. These include definitions of PKE and signature schemes, (one-time/strong) EUF-CMA security, IND-CPA security, (chameleon) hash functions, and the DCR, DDH, and DLIN assumptions.

Key-unique SKE Schemes. A secret-key encryption (SKE) scheme (E,D) consists of two PPT algorithms. Encryption $\mathrm{E}(K, M)$ takes a key $K$ and a message $M$, and outputs a ciphertext $C$. Decryption $\mathrm{D}(K, C)$ takes a key $K$ and a ciphertext $C$, and outputs a message $M$. For correctness, we want $\mathrm{D}(K, C)=M$ for all $M$, all $K$, and all $C \leftarrow \mathrm{E}(K, M)$. We say that (E, D) is key-unique if for every ciphertext $C$, there is at most one key $K$ with $\mathrm{D}(K, C) \neq \perp$. For instance, ElGamal encryption can be interpreted as a key-unique SKE scheme through $\mathrm{E}(x, M):=\left(g^{x}, g^{y}, g^{x y} \cdot M\right)$ (and the obvious D). This example assumes a publicly known group $\mathbb{G}=\langle g\rangle$ in which the $\mathrm{DDH}$ assumption holds 5 If a larger message space (e.g., $\{0,1\}^{*}$ ) is desired, hybrid encryption techniques (which are easily seen to preserve key-uniqueness) can be employed.

Pairings. A (symmetric) pairing is a map $e: \mathbb{G} \times \mathbb{G} \rightarrow \mathbb{G}_{T}$ between two cyclic groups $\mathbb{G}$ and $\mathbb{G}_{T}$ that satisfies $e(g, g) \neq 1$ and $e\left(g^{a}, g^{b}\right)=e(g, g)^{a b}$ for all generators $g$ of $\mathbb{G}$ and all $a, b \in \mathbb{Z}$.

Waters signatures. In [28], Waters proves the following signature scheme EUF-CMA secure 6

- Gen $\left(1^{k}\right)$ chooses groups $\mathbb{G}, \mathbb{G}_{T}$ of prime order $p$, along with a pairing $e: \mathbb{G} \times$ $\mathbb{G} \rightarrow \mathbb{G}_{T}$, a generator $g \in \mathbb{G}$, and uniform group elements $g^{\omega}, H_{0}, \ldots, H_{k} \in$ $\mathbb{G}$. Output is $v k=\left(\mathbb{G}, \mathbb{G}_{T}, e, p, g,\left(H_{i}\right)_{i=0}^{k}, e(g, g)^{\omega}\right)$ and $\operatorname{sigk}=\left(v k, g^{\omega}\right)$.

- $\operatorname{Sig}(\operatorname{sigk}, M)$, for $M=\left(M_{i}\right)_{i=1}^{k} \in\{0,1\}^{k}$, picks $r \leftarrow \mathbb{Z}_{p}$, and outputs $\sigma:=$ $\left(g^{r}, g^{\omega} \cdot\left(H_{0} \prod_{i=1}^{k} H_{i}^{M_{i}}\right)^{r}\right)$.

- $\operatorname{Ver}\left(v k, M,\left(\sigma_{0}, \sigma_{1}\right)\right)$, outputs 1 iff $e\left(g, \sigma_{1}\right)=e(g, g)^{\omega} \cdot e\left(\sigma_{0}, H_{0} \prod_{i=1}^{k} H_{i}^{M_{i}}\right)$.

KDM-CCA and CIRC-CCA security. Let $n=n(k)$ and let PKE be a PKE scheme with message space $\mathcal{M}$. PKE is chosen-ciphertext secure under key-dependent message attacks ( $n$-KDM-CCA secure) iff

$$
\operatorname{Adv}_{\mathrm{PKE}, n, A}^{\mathrm{kdm}-\mathrm{cca}}(k):=\operatorname{Pr}\left[\operatorname{Exp}_{\mathrm{PKE}, n, A}^{\mathrm{kdm}-\mathrm{cca}}(k)=1\right]-1 / 2
$$

is negligible for all $\operatorname{PPT} A$, where experiment $\operatorname{Exp}_{\mathrm{PKE}, n, A}^{\mathrm{kdm}-\mathrm{cca}}$ is defined as follows. First, the experiment tosses a coin $b \leftarrow\{0,1\}$, and samples public parameters $p p \leftarrow \operatorname{Pars}\left(1^{k}\right)$ and $n$ keypairs $\left(p k_{i}, s k_{i}\right) \leftarrow \operatorname{Gen}(p p)$. Then $A$ is invoked with input $p p$ and $\left(p k_{i}\right)_{i=1}^{n}$, and access to two oracles:

- a KDM oracle $\mathcal{K} \mathcal{D} \mathcal{M}_{b}(\cdot, \cdot)$ that maps $i \in[n]$ and a function $f:\left(\{0,1\}^{*}\right)^{n} \rightarrow$ $\{0,1\}^{*}$ to a ciphertext $C \leftarrow \operatorname{Enc}\left(p p, p k_{i}, M\right)$. If $b=0$, then $M=f\left(\left(s k_{i}\right)_{i=1}^{n}\right)$; else, $M=0^{\left|f\left(\left(s k_{i}\right)_{i=1}^{n}\right)\right|}$.

${ }^{5}$ In our application, $\mathbb{G}$ can be made part of the public parameters.

${ }^{6}$ In fact, our description is a slight folklore variant of Waters's scheme. The original scheme features elements $g^{\alpha}, g^{\beta}$ in $v k$, so that $e\left(g^{\alpha}, g^{\beta}\right)$ takes the role of $e(g, g)^{\omega}$. 
- a decryption oracle $\mathcal{D E C}(\cdot, \cdot)$ that takes as input an index $i \in[n]$ and a ciphertext $C$, and outputs $\operatorname{Dec}\left(p p, s k_{i}, C\right)$.

When $A$ finally generates an output $b^{\prime} \in\{0,1\}$, the experiment outputs 1 if $b=b^{\prime}$ (and 0 else). We require that (a) $A$ never inputs a ciphertext $C$ to $\mathcal{D E C}$ that has been produced by $\mathcal{K} \mathcal{D} \mathcal{M}_{b}$ (for the same index $i$ ), and (b) $A$ only specifies PPTcomputable functions $f$ that always output messages of the same length. As a relevant special case, PKE is $n$-CIRC-CCA-secure if it is $n$-KDM-CCA secure against all $A$ that only query $\mathcal{K} \mathcal{D} \mathcal{M}_{b}$ with functions $f \in \mathcal{F}$ for

$$
\mathcal{F}:=\left\{f_{j}: f_{j}\left(\left(s k_{i}\right)_{i=1}^{n}\right)=s k_{j}\right\}_{j \in[n]} \cup\left\{f_{M}: f_{M}\left(\left(s k_{i}\right)_{i=1}^{n}\right)=M\right\}_{M \in \mathcal{M}} .
$$

(Technically, what we call "circular security" is called "clique security" in [10]. However, our notion of circular security implies that of [10].) Our main result will be a PKE scheme that is $n$-CIRC-CCA-secure for all polynomials $n=n(k)$.

\section{$3 \quad$ Lossy Algebraic Filters}

Informal Description. An $\left(\ell_{\mathrm{LAF}}, \mathfrak{n}\right)$-lossy algebraic filter (LAF) is a family of functions indexed by a public key $F p k$ and a tag $t$. A function $\operatorname{LAF}_{F p k, t}$ from the family maps an input $X=\left(X_{i}\right)_{i=1}^{\mathfrak{n}} \in \mathbb{Z}_{p}^{\mathfrak{n}}$ to an output $\operatorname{LAF}_{F p k, t}(X)$, where $p$ is

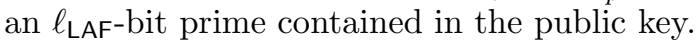

The crucial property of an LAF is its lossiness. Namely, for a given public key $F p k$, we distinguish injective and lossy tags 7 For an injective tag $t$, the function $\operatorname{LAF}_{F p k, t}(\cdot)$ is injective, and thus has an image of size $p^{\mathfrak{n}}$. However, if $t$ is lossy, then $\operatorname{LAF}_{F p k, t}(\cdot)$ only depends on a linear combination $\sum_{i=1}^{\mathfrak{n}} \omega_{i} X_{i} \bmod p$ of its input. In particular, different $X$ with the same value $\sum_{i=1}^{\mathfrak{n}} \omega_{i} X_{i}$ mod $p$ are mapped to the same image. Here, the coefficients $\omega_{i} \in \mathbb{Z}_{p}$ only depend on $F p k$ (but not on $t$ ). For a lossy tag $t$, the image of $\operatorname{LAF}_{F p k, t}(\cdot)$ is thus of size at most $p$. Note that the modulus $p$ is public, while the coefficients $\omega_{i}$ may be (and in fact will have to be) computationally hidden.

For this concept to be useful, we require that (a) lossy and injective tags are computationally indistinguishable, (b) lossy tags can be generated using a special trapdoor, but (c) new lossy (or, rather, non-injective) tags cannot be found efficiently without that trapdoor, even when having seen polynomially many lossy tags before. In view of our application, we will work with structured tags: each tag $t=\left(t_{\mathrm{c}}, t_{\mathrm{a}}\right)$ consists of a core tag $t_{\mathrm{c}}$ and an auxiliary tag $t_{\mathrm{a}}$. The auxiliary tag will be a ciphertext part that is authenticated by a filter image.

Definition 1. An ( $\left.\ell_{\mathrm{LAF}}, \mathfrak{n}\right)$-lossy algebraic filter (LAF) LAF consists of three PPT algorithms:

Key generation. FGen $\left(1^{k}\right)$ samples a keypair $(F p k, F t d)$. The public key Fpk contains an $\ell_{\mathrm{LAF}}-$ bit prime $p$ and the description of a tag space $\mathcal{T}=\mathcal{T}_{\mathrm{c}} \times$ $\{0,1\}^{*}$ for efficiently samplable $\mathcal{T}_{\mathrm{c}}$. A tag $t=\left(t_{\mathrm{c}}, t_{\mathrm{a}}\right)$ consists of a core tag $t_{\mathrm{c}} \in \mathcal{T}_{\mathrm{c}}$ and an auxiliary tag $t_{\mathrm{a}} \in\{0,1\}^{*}$. A tag may be injective, or lossy, or neither. Ftd is a trapdoor that will allow to sample lossy tags.

\footnotetext{
7 Technically, there may also be tags that are neither injective nor lossy.
} 
Evaluation. FEval $(F p k, t, X)$, for a public key Fpk and a tag $t=\left(t_{\mathrm{c}}, t_{\mathrm{a}}\right) \in \mathcal{T}$, maps an input $\left.X=\left(X_{i}\right)_{i=1}^{\mathfrak{n}}\right) \in \mathbb{Z}_{p}^{\mathfrak{n}}$ to a unique output $\operatorname{LAF}_{F p k, t}(X)$.

Lossy tag generation. FTag $\left(F t d, t_{\mathrm{a}}\right)$, for a trapdoor Ftd and $t_{\mathrm{a}} \in\{0,1\}^{*}$, samples a core tag $t_{\mathrm{c}}$ such that $t=\left(t_{\mathrm{c}}, t_{\mathrm{a}}\right)$ is lossy.

We require the following:

Lossiness. The function $\operatorname{LAF}_{F p k, t}(\cdot)$ is injective if $t$ is injective. If $t$ is lossy, then $\operatorname{LAF}_{F p k, t}(X)$ depends only on $\sum_{i=1}^{\mathfrak{n}} \omega_{i} X_{i} \bmod p$ for $\omega_{i} \in \mathbb{Z}_{p}$ that only depend on Fpk.

Indistinguishability. Lossy tags are indistinguishable from random tags:

$$
\operatorname{Adv}_{\mathrm{LAF}, A}^{\text {ind }}(k):=\operatorname{Pr}\left[A\left(1^{k}, F p k\right)^{\mathrm{FTag}(F t d, \cdot)}=1\right]-\operatorname{Pr}\left[A\left(1^{k}, F p k\right)^{\mathcal{O}_{\mathcal{T}_{c}}(\cdot)}=1\right]
$$

is negligible for all PPT A, where $(F p k, F t d) \leftarrow \mathrm{FGen}\left(1^{k}\right)$, and $\mathcal{O}_{\mathcal{T}_{\mathrm{c}}}(\cdot)$ is the oracle that ignores its input and samples a random core tag $t_{\mathrm{c}}$.

Evasiveness. Non-injective (and in particular lossy) tags are hard to find, even given multiple lossy tags:

$$
\operatorname{Adv}_{\mathrm{LAF}, A}^{\mathrm{eva}}(k):=\operatorname{Pr}\left[t \text { non-injective } \mid t \leftarrow A\left(1^{k}, F p k\right)^{\mathrm{FTag}(F t d, \cdot)}\right]
$$

is negligible with $(F p k, F t d) \leftarrow \mathrm{FGen}\left(1^{k}\right)$, and for any PPT algorithm $A$ that never outputs a tag obtained through oracle queries (i.e., A never outputs $t=\left(t_{\mathrm{c}}, t_{\mathrm{a}}\right)$ when $t_{\mathrm{c}}$ has been obtained by an oracle query $\left.\mathrm{FTag}\left(F t d, t_{\mathrm{a}}\right)\right)$.

\subsection{Construction}

Intuition. We present a construction based on the DLIN problem in a group $\mathbb{G}$ of order $p$ with symmetric pairing $e: \mathbb{G} \times \mathbb{G} \rightarrow \mathbb{G}_{T}$. Essentially, each tag corresponds to $\mathfrak{n}$ DLIN-encrypted Waters signatures. If the signatures are valid, the tag is lossy. The actual filter maps an input $X=\left(X_{i}\right)_{i=1}^{\mathfrak{n}} \in \mathbb{Z}_{p}^{\mathfrak{n}}$ to the tuple

$$
\operatorname{LAF}_{F p k, t}(X):=\mathbf{M} \circ X:=\left(\prod_{j=1}^{\mathfrak{n}} \mathbf{M}_{i, j}^{X_{j}}\right)_{j=1}^{\mathfrak{n}} \in \mathbb{G}_{T}^{\mathfrak{n}},
$$

where the matrix $\mathbf{M}=\left(\mathbf{M}_{i, j}\right)_{i, j \in[\mathfrak{n}]} \in \mathbb{G}_{T}^{\mathfrak{n} \times \mathfrak{n}}$ is computed from public key and tag. Note that this mapping is lossy if and only if the matrix

$$
\widetilde{\mathbf{M}}:=\left(\widetilde{\mathbf{M}}_{i, j}\right):=\left(\operatorname{dlog}_{e(g, g)}\left(\mathbf{M}_{i, j}\right)\right)_{i, j} \in \mathbb{Z}_{p}^{\mathfrak{n} \times \mathfrak{n}}
$$

of discrete logarithms (to some arbitrary basis $e(g, g) \in \mathbb{G}_{T}$ ) is non-invertible.

For a formal description, let $\ell_{\mathrm{LAF}}(k), \mathfrak{n}(k)$ be two functions.

Key generation. FGen $\left(1^{k}\right)$ generates cyclic groups $\mathbb{G}, \mathbb{G}_{T}$ of prime order $p$ (where $p$ has bitlength $\left\lfloor\log _{2}(p)\right\rfloor=\ell_{\mathrm{LAF}}(k)$ ), and a symmetric pairing $e$ : $\mathbb{G} \times \mathbb{G} \rightarrow \mathbb{G}_{T}$. Then FGen chooses

- a generator $g \in \mathbb{G}$ and a uniform exponent $\omega \leftarrow \mathbb{Z}_{p}$,

- uniform group elements $U_{1}, \ldots, U_{\mathfrak{n}} \leftarrow \mathbb{G}, H_{0}, \ldots, H_{k} \leftarrow \mathbb{G}$, and

- a keypair $(H p k, H t d)$ for a chameleon hash $\mathrm{CH}:\{0,1\}^{*} \rightarrow\{0,1\}^{k}$. 
FGen finally outputs

$$
\begin{aligned}
F p k & :=\left(\mathbb{G}, \mathbb{G}_{T}, e, p, g,\left(H_{i}\right)_{i=0}^{k},\left(U_{i}\right)_{i=1}^{\mathfrak{n}}, W:=e(g, g)^{\omega}, H p k\right) \\
F t d & :=\left(F p k, g^{\omega}, H t d\right) .
\end{aligned}
$$

For convenience, write $U_{i}=g^{u_{i}}$ for suitable (unknown) exponents $u_{i}$. Tags. (Core) tags are of the form

$$
t_{\mathrm{c}}:=\left(R,\left(\widetilde{S}_{i}\right)_{i=1}^{\mathfrak{n}},\left(S_{i, j}\right)_{i, j=1}^{\mathfrak{n}}, R_{\mathrm{CH}}\right) \in \mathbb{G} \times \mathbb{G} \times \mathbb{G}^{\mathfrak{n} \times \mathfrak{n}} \times \mathcal{R}_{\mathrm{CH}}
$$

(for CH's randomness space $\mathcal{R}_{\mathrm{CH}}$ ), where we require $e\left(U_{j^{\prime}}, S_{i, j}\right)=e\left(U_{j}, S_{i, j^{\prime}}\right)$ whenever $i \notin\left\{j, j^{\prime}\right\}$. This means we can write $R=g^{r}, \widetilde{S_{i}}=g^{\widetilde{s_{i}}}$, and $S_{i, j}=$ $U_{j}^{s_{i}}$ (for $i \neq j$ ) for suitable $r, s_{i}, \widetilde{s}_{i}$. To a tag $t=\left(t_{\mathrm{c}}, t_{\mathrm{a}}\right.$ ) (with auxiliary part $\left.t_{\mathrm{a}} \in\{0,1\}^{*}\right)$, we associate the matrix $\mathbf{M}=\left(\mathbf{M}_{i, j}\right)_{i, j=1}^{\mathfrak{n}} \in \mathbb{G}_{T}^{\mathfrak{n} \times \mathfrak{n}}$ with

$$
\begin{aligned}
& \mathbf{M}_{i, j}=e\left(U_{j}, \widetilde{S}_{i}\right) \cdot e\left(g, S_{i, j}\right)=e(g, g)^{u_{j}\left(\widetilde{s_{i}}+s_{i}\right)} \quad(i \neq j) \\
& \mathbf{M}_{i, i}=\frac{e\left(g, S_{i, i}\right)}{W \cdot e\left(H_{0} \prod_{i=1}^{k} H_{i}^{T_{i}}, R\right)}
\end{aligned}
$$

for $\left(T_{i}\right)_{i=1}^{k}:=\mathrm{CH}_{H p k}\left(R,\left(\widetilde{S}_{i}\right)_{i=1}^{\mathfrak{n}},\left(S_{i, j}\right)_{i, j=1}^{\mathfrak{n}}, t_{\mathrm{a}} ; R_{\mathrm{CH}}\right)$. If the matrix $\widetilde{\mathbf{M}}$ of discrete logarithms (see (2)) is invertible, we say that $t$ is injective; if $\widetilde{\mathbf{M}}$ has rank 1 , then $t$ is lossy. Thus, for lossy tags, $\mathbf{M}_{i, j}=e(g, g)^{u_{j}\left(\widetilde{s_{i}}+s_{i}\right)}$ for all $i, j$.

Evaluation. FEval $(F p k, t, X)$, for $t=\left(t_{\mathrm{c}}, t_{\mathrm{a}}\right), t_{\mathrm{a}} \in\{0,1\}^{*}, X=\left(X_{i}\right)_{i=1}^{\mathfrak{n}} \in$ $\mathbb{Z}_{p}^{\mathfrak{n}}$, and $F p k$ and $t_{\mathrm{c}}$ as above, computes $\mathbf{M}$ as in (3) and then $\left(Y_{i}\right)_{i=1}^{\mathfrak{n}}:=$ $\operatorname{LAF}_{F p k, t}(X) \in \mathbb{G}_{T}^{\mathfrak{n}}$ as in (11).

Lossiness. If we write $Y_{i}=e(g, g)^{y_{i}}$, the definition of FEval implies $\left(y_{i}\right)_{i=1}^{\mathfrak{n}}=$ $\widetilde{\mathbf{M}} \cdot X$. Since injective tags satisfy that $\widetilde{\mathbf{M}}$ is invertible, they lead to injective functions $\operatorname{LAF}_{F p k, t}(\cdot)$. But for a lossy tag, $\widetilde{\mathbf{M}}_{i, j}=u_{j}\left({\widetilde{s_{i}}}+s_{i}\right)$, so that

$$
y_{i}=\sum_{j=1}^{\mathfrak{n}} u_{j}\left(\widetilde{s_{i}}+s_{i}\right) X_{j}=\left(\widetilde{s_{i}}+s_{i}\right) \cdot \sum_{j=1}^{\mathfrak{n}} u_{j} X_{j} \quad \bmod p .
$$

Specifically, $\operatorname{LAF}_{F p k, t}(X)$ depends only on $\sum_{i} \omega_{i} X_{i} \bmod p$ for $\omega_{i}:=u_{i}$.

Lossy tag generation. $\mathrm{FTag}\left(F t d, t_{\mathrm{a}}\right)$, for $F t d$ as above and $t_{\mathrm{a}} \in\{0,1\}^{*}$, first chooses a random $\mathrm{CH}$-image $T=\left(T_{i}\right)_{i=1}^{k} \in\{0,1\}^{k}$ that can later be explained, using $\mathrm{Htd}$, as the $\mathrm{CH}$-image of an arbitrary preimage. FTag then chooses uniform $r, s_{i}, \widetilde{s}_{i} \leftarrow \mathbb{Z}_{p}$ and sets (for $i \neq j$ )

$$
\begin{aligned}
R & :=g^{r}, \quad \widetilde{S_{i}}:=g^{\widetilde{s_{i}}}, \\
S_{i, j} & :=U_{j}^{s_{i}}, \quad S_{i, i}:=U_{i}^{\widetilde{s_{i}}+s_{i}} \cdot g^{\omega} \cdot\left(H_{0} \prod_{i=1}^{k} H_{i}^{T_{i}}\right)^{r} .
\end{aligned}
$$

Finally, FTag chooses $R_{\mathrm{CH}}$ with $\mathrm{CH}_{H p k}\left(R,\left(\widetilde{S}_{i}\right)_{i=1}^{\mathfrak{n}},\left(S_{i, j}\right)_{i, j=1}^{\mathfrak{n}}, t_{\mathrm{a}} ; R_{\mathrm{CH}}\right)=T$ and outputs $t_{\mathrm{c}}=\left(R,\left(\widetilde{S}_{i}\right)_{i=1}^{\mathfrak{n}},\left(S_{i, j}\right)_{i, j=1}^{\mathfrak{n}}, R_{\mathrm{CH}}\right)$. Intuitively, $t_{\mathrm{c}}$ consists of $\mathfrak{n}$ DLIN encryptions (with correlated randomness $s_{i}, \widetilde{s}_{i}$ ) of Waters signatures $\left(g^{r}, g^{\omega} \cdot\left(H_{0} \prod_{i=1}^{k} H_{i}^{T_{i}}\right)^{r}\right)$ for message $T$. Indeed, substituting into (3) yields 


$$
\mathbf{M}_{i, i}:=\frac{e(g, g)^{u_{i}\left(\widetilde{s_{i}}+s_{i}\right)} \cdot W \cdot e\left(g,\left(H_{0} \prod_{i=1}^{k} H_{i}^{T_{i}}\right)^{r}\right)}{W \cdot e\left(g^{r}, H_{0} \prod_{i=1}^{k} H_{i}^{T_{i}}\right)}=e(g, g)^{u_{i}\left(\widetilde{s_{i}}+s_{i}\right)} .
$$

Hence, $\widetilde{\mathbf{M}}_{i, j}=u_{j}\left(\widetilde{s}_{i}+s_{i}\right)$ for all $i, j$, and thus the resulting tag $t=\left(t_{\mathrm{c}}, t_{\mathrm{a}}\right)$ is lossy.

A Generalization. In the full paper [21], we also show how to generalize the above construction to achieve constant-size tags and evaluation keys.

Other Instances and Further Applications of LAFs. Since LAFs can be seen as "disguised signature schemes", it seems interesting to try to convert other signature schemes (and in particular schemes that do not require pairing-friendly groups) to LAFs. Besides, LAFs would seem potentially useful in other settings, specifically in settings with inherently many challenges (e.g., the selective-opening setting [6]).

Theorem 1. If the DLIN assumption holds in $\mathbb{G}$, and $\mathrm{CH}$ is a chameleon hash function, then the LAF construction LAF from Section 3.1 satisfies Definition 1.

The lossiness of LAF has already been discussed in Section 3.1. We prove indistinguishability and evasiveness separately.

Lemma 1. For every adversary $A$ on LAF's indistinguishability, there exists a DLIN distinguisher $B$ such that $\operatorname{Adv}_{\mathrm{LAF}, A}^{\text {ind }}(k)=\mathfrak{n} \cdot \operatorname{Adv}_{B}^{\operatorname{dlin}}(k)$.

Intuitively, to see Lemma 1, observe that lossy tags differ from random tags only in their $S_{i, i}$ components, and in how the $\mathrm{CH}$ randomness $R_{\mathrm{CH}}$ is generated. For lossy tags, the $S_{i, i}$ are (parts of) DLIN ciphertexts, which are pseudorandom under the DLIN assumption. Furthermore, the uniformity property of $\mathrm{CH}$ guarantees that the distribution of $R_{\mathrm{CH}}$ is the same for lossy and random tags. We formally prove Lemma 1] in the full version 21].

Lemma 2. For every adversary $A$ on LAF's evasiveness, there exist adversaries $B, C$, and $F$ with $\operatorname{Adv}_{\mathrm{LAF}, A}^{\text {eva }}(k) \leq\left|\operatorname{Adv}_{\mathrm{LAF}, B}^{\text {ind }}(k)\right|+\operatorname{Adv}_{\mathrm{CH}, C}^{\mathrm{cr}}(k)+\operatorname{Adv}_{\mathrm{Sig}_{\text {wat }}^{\text {euf }}, F}^{\text {ecma }}(k)$.

Intuitively, Lemma 2 holds because lossy (or, rather, non-injective) tags correspond to DLIN-encrypted Waters signatures. Hence, even after seeing many lossy tags (i.e., encrypted signatures), an adversary cannot produce a fresh encrypted signature. We note that the original Waters signatures from [28] are re-randomizable and thus not strongly unforgeable. To achieve evasiveness, we have thus used a chameleon hash function, much like Boneh et al. [8] did to make Waters signatures strongly unforgeable. We give a formal proof in [21].

Combining Lemma 1, Lemma 2, and the fact that Waters signatures are EUFCMA secure already under the $\mathrm{CDH}$ assumption, we obtain Theorem 1.

\section{CIRC-CCA-Secure Encryption Scheme}

Setting and Ingredients. First, we assume an algorithm GenN that outputs $\ell_{N}$-bit Blum integers $N=P Q$ along with their prime factors $P$ and $Q$. If $N$ 
is clear from the context, we write $\mathbb{G}_{\mathrm{rnd}}$ and $\mathbb{G}_{\mathrm{msg}}$ for the unique subgroups of $\mathbb{Z}_{N^{3}}^{*}$ of order $(P-1)(Q-1) / 4$, resp. $N^{2}$. We also write $h:=1+N \bmod N^{3}$, so $\langle h\rangle=\mathbb{G}_{\mathrm{msg}}$. Note that it is efficiently possible to compute $\operatorname{dlog}_{h}(X):=x$ for $X:=h^{x} \in \mathbb{G}_{\mathrm{msg}}$ and $x \in \mathbb{Z}_{N^{2}}$. Specifically, it is efficiently possible to test for membership in $\mathbb{G}_{\mathrm{msg}}$. In our scheme, $\mathbb{G}_{\mathrm{msg}}$ will be used to embed a suitably encoded message, and $\mathbb{G}_{\text {rnd }}$ will be used for blinding. We require that

- $P$ and $Q$ are safe primes of bitlength between $\ell_{N} / 2-k$ and $\ell_{N} / 2+k$,

$-\operatorname{gcd}((P-1)(Q-1) / 4, N)=1$ (as, e.g., for uniform $P, Q$ of a certain length),

$-\ell_{N} \geq 25 k+8$ (e.g., $k=80$ and $\left.\ell_{N}=2048\right)$

- the DCR assumption holds in $\mathbb{Z}_{N^{3}}^{*}$, and the DDH assumption holds in $\mathbb{G}_{\mathrm{rnd}}$.

We also assume an $\left(\ell_{\text {LAF }}, \mathfrak{n}\right)$-lossy algebraic filter LAF for $\mathfrak{n}=6$ and $\ell_{\text {LAF }}=$ $\left(\ell_{N}+k+1\right) /(\mathfrak{n}-2)$. Our scheme will encrypt messages from the domain

$$
\mathcal{M}:=\mathbb{Z}_{2^{3 k}} \times \mathbb{Z}_{p \cdot 2^{k}} \times \mathbb{Z}_{N \cdot 2^{k-2}},
$$

where $p$ is the modulus of the used LAF. (The reason for this weird-looking message space will become clearer in the proof.) During encryption, we will have to treat a message $M=(a, b, c) \in \mathcal{M}$ both as an element of $\mathbb{Z}_{N^{2}}$ and as an LAF-input from $\mathbb{Z}_{p}^{\mathfrak{n}}$. In these cases, we can encode

$$
\begin{aligned}
\mathbb{Z} & :=a+2^{3 k} \cdot b+p \cdot 2^{4 k} \cdot c \in \mathbb{Z}, \\
{[M]_{\mathbb{Z}_{p}^{\mathfrak{n}}} } & :=\left(a, b \bmod p, c_{0}, \ldots, c_{\mathfrak{n}-3}\right) \in \mathbb{Z}_{p}^{\mathfrak{n}}
\end{aligned}
$$

for the natural interpretation of $\mathbb{Z}_{i}$-elements as integers between 0 and $i-1$, and c's $p$-adic representation $\left(c_{i}\right)_{i=0}^{\mathfrak{n}-3} \in \mathbb{Z}_{p}^{\mathfrak{n}-2}$ with $c=\sum_{i=0}^{\mathfrak{n}-3} c_{i} \cdot p^{i}$. By our choice of $\ell_{N}$ and $\ell_{\text {LAF }}$, we have $0 \leq[M]_{\mathbb{Z}}<N^{2}-2^{k}$. However, the encoding $[M]_{\mathbb{Z}_{p}^{\text {n }}}$ is not injective, since it only depends on $b \bmod p\left(\right.$ while $0 \leq b<p \cdot 2^{k}$ ).

Finally, we assume a strongly OT-EUF-CMA secure signature scheme Sig $=$ (SGen, Sig, Ver) with $k$-bit verification keys, and a key-unique IND-CPA secure symmetric encryption scheme (E,D) (see Section 2) with $k$-bit symmetric keys $K$ and message space $\{0,1\}^{*}$.

Now consider the following PKE scheme PKE:

Public Parameters. Pars $\left(1^{k}\right)$ first runs $(N, P, Q) \leftarrow \operatorname{GenN}\left(1^{k}\right)$. Recall that this fixes the groups $\mathbb{G}_{\mathrm{rnd}}$ and $\mathbb{G}_{\mathrm{mgg}}$. Then, Pars selects two generators $g_{1}, g_{2}$ of $\mathbb{G}_{\mathrm{rnd}}$. Finally, Pars runs $(F p k, F t d) \leftarrow \mathrm{FGen}\left(1^{k}\right)$ and outputs $p p=\left(N, g_{1}, g_{2}, F p k\right)$. In the following, we denote with $p$ the LAF modulus contained in $F p k$.

Key Generation. Gen $(p p)$ uniformly selects four messages $s_{j}=\left(a_{j}, b_{j}, c_{j}\right) \in$ $\mathcal{M}($ for $1 \leq j \leq 4)$ as secret key, and sets $p k:=\left(u:=g_{1}^{\left[s_{1}\right]_{\mathbb{Z}}} g_{2}^{\left[s_{2}\right]_{\mathbb{Z}}}, v:=g_{1}^{\left[s_{3}\right]_{\mathbb{Z}}} g_{2}^{\left[s_{4}\right]_{\mathbb{Z}}}\right)$ and $s k:=\left(s_{j}\right)_{j=1}^{4}$.

Encryption. $\operatorname{Enc}(p p, p k, M)$, for $p p$ and $p k$ as above, and $M \in \mathcal{M}$, uniformly selects exponents $r, \widetilde{r} \leftarrow \mathbb{Z}_{N / 4}$, a random filter core tag $t_{\mathrm{c}}$, a Sig-keypair $(v k, s i g k) \leftarrow \mathrm{SGen}\left(1^{k}\right)$, and a random symmetric key $K \in\{0,1\}^{k}$ for $(\mathrm{E}, \mathrm{D})$, and computes 


$$
\begin{aligned}
\left(G_{1}, G_{2}\right) & :=\left(g_{1}^{r}, g_{2}^{r}\right) & & C_{\mathrm{E}} \leftarrow \mathrm{E}\left(K, \operatorname{LAF}_{F p k, t}\left([M]_{\mathbb{Z}_{p}^{\mathfrak{n}}}\right)\right) \\
\left(\widetilde{G}_{1}, \widetilde{G}_{2}\right) & :=\left(g_{1}^{\widetilde{r}}, g_{2}^{\widetilde{r}}\right) & & \sigma \leftarrow \operatorname{Sig}\left(\operatorname{sigk},\left(\left(G_{j}, \widetilde{G}_{j}\right)_{j=1}^{2}, Z, \widetilde{Z}, C_{\mathrm{E}}, t_{\mathrm{c}}\right)\right) \\
Z & :=\left(u^{v k} v\right)^{r \cdot N^{2}} & & C:=\left(\left(G_{j}, \widetilde{G}_{j}\right)_{j=1}^{2}, Z, \widetilde{Z}, C_{\mathrm{E}}, t_{\mathrm{c}}, v k, \sigma\right) \\
\widetilde{Z} & :=\left(u^{v k} v\right)^{r} u^{\widetilde{r}} h^{K+2^{k} \cdot[M]_{\mathbb{Z}}} & &
\end{aligned}
$$

for the auxiliary tag $t_{\mathrm{a}}:=v k$, and the resulting filter tag $t:=\left(t_{\mathrm{c}}, t_{\mathrm{a}}\right)$.

Decryption. $\operatorname{Dec}(p p, s k, C)$, for $p p$, sk and $C$ as above, first checks the signature $\sigma$ and rejects with $\perp$ if $\operatorname{Ver}\left(v k,\left(\left(G_{j}, \widetilde{G}_{j}\right)_{j=1}^{2}, Z, \widetilde{Z}, C_{\mathrm{E}}, t_{\mathrm{c}}\right), \sigma\right)=0$, or if

$$
Z \neq\left(G_{1}^{\left[s_{1}\right]_{\mathbb{Z}} \cdot v k+\left[s_{3}\right]_{\mathbb{Z}}} G_{2}^{\left[s_{2}\right]_{\mathbb{Z}} \cdot v k+\left[s_{4}\right]_{\mathbb{Z}}}\right)^{N^{2}}
$$

Then Dec computes

$$
Z^{\prime}:=G_{1}^{\left[s_{1}\right]_{\mathbb{Z}} \cdot v k+\left[s_{3}\right]_{\mathbb{Z}}} G_{2}^{\left[s_{2}\right]_{\mathbb{Z}} \cdot v k+\left[s_{4}\right]_{\mathbb{Z}}} \widetilde{G}_{1}^{\left[s_{1}\right]_{\mathbb{Z}}} \widetilde{G}_{2}^{\left[s_{2}\right]_{\mathbb{Z}}}
$$

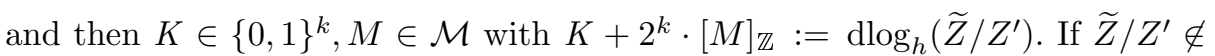
$\mathbb{G}_{\mathrm{msg}}$, or no such $M$ exists, or $\mathrm{D}\left(K, C_{\mathrm{E}}\right) \neq \operatorname{LAF}_{F p k, t}\left([M]_{\mathbb{Z}_{p}^{\mathfrak{n}}}\right)\left(\right.$ for $t=\left(t_{\mathrm{c}}, t_{\mathrm{a}}\right)$ computed as during encryption), then Dec rejects with $\perp$. Else, Dec outputs $M$.

Secret Keys as Messages. Our scheme has secret keys $s=\left(s_{j}\right)_{j=1}^{4} \in \mathcal{M}^{4}$; hence, we can only encrypt one quarter $s_{j}$ of a secret key at a time. In the security proof below, we will thus only consider KDM queries that ask to encrypt a specific secret key part. Alternatively, we can change our scheme, so that 4tuples of $\mathcal{M}$-elements are encrypted. To avoid malleability (which would destroy CCA security), we of course have to use only one LAF tag for this. Our CIRCCCA proof below applies to such a changed scheme with minor syntactic changes.

Efficiency. When instantiated with our DLIN-based LAF construction from Section 3, and taking $\mathfrak{n}=6$ as above, our scheme has ciphertexts with $43 \mathbb{G}$ -

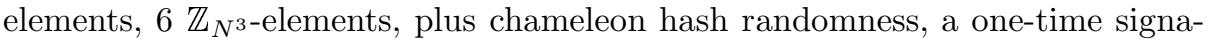
ture and verification key, and a symmetric ciphertext (whose size could be in

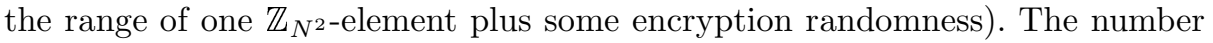
of group elements in the ciphertext is constant, and does not grow in the security parameter. The public parameters contain $\mathbf{O}(k)$ group element: 8 (most of

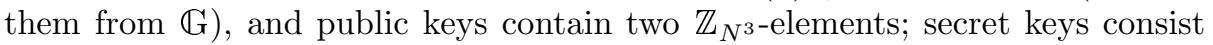

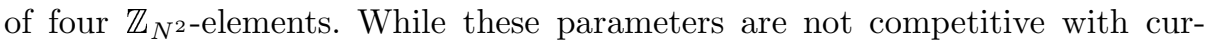
rent non-KDM-secure schemes, they are significantly better than those from the circular-secure scheme of Camenisch et al. [14] 9

Security Proof (single-user user). It is instructive to first treat the singleuser case. Here, we essentially only require that PKE is IND-CCA secure, even if

\footnotetext{
${ }^{8}$ Using the generalized LAF mentioned at the end of Section 3.1 public parameters with $\mathbf{O}(1)$ group elements are possible, at the cost of a (constant) number of extra group elements per tag.

9 For instance, Section 7 of the full version of [14] implies that their scheme has a public key, resp. ciphertext of about 500, resp. $1000 \mathbb{G}$-elements (for $\log _{2}(|\mathbb{G}|)=160$ ).
} 
encryptions of its secret key are made public. For the multi-user case (see [21]), we can then proceed like [10, 11] and re-randomize keys and ciphertexts of a single PKE instance. This enables an analysis analogous to the single-user case.

Theorem 2. Assume the DCR assumption holds in $\mathbb{Z}_{N^{3}}$, the DDH assumption holds in $\mathbb{G}_{\mathrm{rnd}}$, LAF is an LAF, Sig is a strongly OT-EUF-CMA secure signature scheme, $\mathrm{H}$ is collision-resistant, and (E, D) is a key-unique IND-CPA secure SKE scheme. Then PKE is 1-CIRC-CCA-secure.

Proof. Assume a PPT adversary $A$ on PKE's 1-CIRC-CCA security. Say that $A$ always makes $q=q(k) \mathrm{KDM}$ queries. We proceed in games. Let out $_{i}$ denote the output of Game $i$.

Game 1 is the 1-KDM-CCA experiment with PKE and $A$. By definition, $\operatorname{Pr}\left[\right.$ out $\left._{1}=1\right]-1 / 2=\operatorname{Adv}_{\mathrm{PKE}, A}^{\mathrm{kdm}-\mathrm{cca}}(k)$.

In Game 2, we modify the way KDM queries are answered. Namely, in each ciphertext prepared for $A$, we set up $Z$ and $\widetilde{Z}$ up as

$$
\begin{aligned}
Z & :=\left(G_{1}^{\left[s_{1}\right]_{\mathbb{Z}} \cdot v k+\left[s_{3}\right]_{\mathbb{Z}}} G_{2}^{\left[s_{2}\right]_{\mathbb{Z}} \cdot v k+\left[s_{4}\right]_{\mathbb{Z}}}\right)^{N^{2}} \\
\widetilde{Z} & :=G_{1}^{\left[s_{1}\right]_{\mathbb{Z}} \cdot v k+\left[s_{3}\right]_{\mathbb{Z}}} G_{2}^{\left[s_{2}\right]_{\mathbb{Z}} \cdot v k+\left[s_{4}\right]_{\mathbb{Z}}} \widetilde{G}_{1}^{\left[s_{1}\right]_{\mathbb{Z}}} \widetilde{G}_{2}^{\left[s_{2}\right]_{\mathbb{Z}}} \cdot h^{K+2^{k} \cdot[M]_{\mathbb{Z}}} .
\end{aligned}
$$

for the already prepared $\left(G_{j}, \widetilde{G}_{j}\right)=\left(g_{j}^{r}, g_{j}^{\widetilde{r}}\right)$. This change is only conceptual by our setup of $u, v$, so $\operatorname{Pr}\left[\right.$ out $\left._{2}=1\right]=\operatorname{Pr}\left[\right.$ out $\left._{1}=1\right]$.

In Game 3, we again change how KDM ciphertexts are prepared. Intuitively, our goal is now to prepare the $G_{j}$ and $\widetilde{G}_{j}$ with additional $\mathbb{G}_{\text {msg-components, }}$ such that $\widetilde{Z}$, as computed in ([6), is of the form $g \cdot h^{K}$ for some $g \in \mathbb{G}_{\text {rnd }}$. (That is, we want the $\mathbb{G}_{\mathrm{msg}}$-components of the $G_{j}, \widetilde{G}_{j}$ to cancel out the $h^{2^{k} \cdot[M]_{\mathbb{Z}}}$ term in (6).) To do so, we prepare $G_{j}=g_{j}^{r} / h^{\alpha_{j} \cdot 2^{k}}$ and $\widetilde{G}_{j}=g_{j}^{r} / h^{\widetilde{\alpha}_{j} \cdot 2^{k}}$ for $j \in\{1,2\}$ and suitable $\alpha_{j}, \widetilde{\alpha}_{j}$ to be determined. $\widetilde{Z}$ is still computed as in (6), so

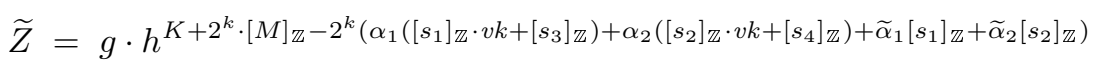

for $g=g_{1}^{r \cdot\left(\left[s_{1}\right]_{\mathbb{Z}} \cdot v k+\left[s_{3}\right]_{\mathbb{Z}}\right)+\widetilde{r}\left[s_{1}\right]_{\mathbb{Z}}} g_{2}^{r \cdot\left(\left[s_{2}\right]_{\mathbb{Z}} \cdot v k+\left[s_{4}\right]_{\mathbb{Z}}\right)+\widetilde{r}\left[s_{2}\right]_{\mathbb{Z}}}=\left(u^{v k} v\right)^{r} u^{\widetilde{r}} \in \mathbb{G}_{\mathrm{rnd}}$. So to prepare a KDM encryption of $s_{j^{*}}$ with $\widetilde{Z}=g \cdot h^{K}$, we can set $\left(\alpha_{1}, \alpha_{2}, \widetilde{\alpha}_{1}, \widetilde{\alpha}_{2}\right)$ to $(0,0,1,0)$ for $j^{*}=1$, to $(1,0,-v k, 0)$ for $j^{*}=2$, to $(0,0,1,0)$ for $j^{*}=3$, and to $(0,1,0,-v k)$ for $j^{*}=4$. ( $v k$ can be chosen independently in advance.) The remaining parts of $C$ are prepared as in Game 2. We claim

$$
\operatorname{Pr}\left[\text { out }_{3}=1\right]-\operatorname{Pr}\left[\text { out }_{2}=1\right] \leq 4 \cdot \operatorname{Adv}_{\mathbb{Z}_{N^{3}}^{*}, B}^{\mathrm{dcr}}(k)+\mathbf{O}\left(2^{-k}\right)
$$

for a suitable DCR distinguisher $B$ that simulates Game 2, resp. Game 3. Concretely, $B$ gets as input a value $\widetilde{W} \in \mathbb{Z}_{N^{3}}^{*}$ of the form $\widetilde{W}=\tilde{g}^{N^{2}} \cdot h^{b}$ for $b \in\{0,1\}$. Note that if we set $W:=\widetilde{W}^{-2^{k}}$, we have $W=g^{\widehat{r}} / h^{b \cdot 2^{k}} \in \mathbb{Z}_{N^{3}}^{*}$, with uniform $g^{\widehat{r}} \in \mathbb{G}_{\text {rnd }}$. First, $B$ guesses a value of $j^{*} \in[4]$. (This gives a very small hybrid argument, in which in the $j^{*}$-th step, only encryptions of $s_{j^{*}}$ are changed.) We 
only detail $B$ 's behavior for the case $j^{*}=3$; the other cases are easier or analogous. First, $B$ sets up $g_{1}:=W^{N^{2}}$ and $g_{2}:=W^{\gamma N^{4}}$ for uniform $\gamma \in \mathbb{Z}_{N / 4}$. To prepare an encryption of $s_{3}, B$ chooses uniform $\rho, \widetilde{\rho} \in \mathbb{Z}_{N^{2} / 4}$ and sets

$$
\begin{array}{ll}
G_{1}:=W^{\rho \cdot\left(\rho^{-1}\right)} & G_{2}:=W^{\gamma \cdot \rho \cdot\left(\rho^{-1}\right) \cdot N^{2}} \\
\widetilde{G}_{1}:=W^{v k \cdot \widetilde{\rho} \cdot\left(\widetilde{\rho}^{-1}\right)} & \widetilde{G}_{2}:=W^{\gamma \cdot v k \cdot \widetilde{\rho} \cdot\left(\widetilde{\rho}^{-1}\right) \cdot N^{2}},
\end{array}
$$

where the values $\rho^{-1}, \widetilde{\rho}^{-1}$ are computed modulo $N^{2}$. This implicitly sets $r=\rho$. $\left(\rho^{-1}\right) / N^{2} \bmod \left|\mathbb{G}_{\mathrm{rnd}}\right|$ and $\widetilde{r}=v k \cdot \widetilde{\rho} \cdot\left(\widetilde{\rho}^{-1}\right) / N^{2} \bmod \left|\mathbb{G}_{\mathrm{rnd}}\right|$, both of which are statistically close to uniform. Furthermore, $G_{j}=g_{j}^{r} / h^{b \cdot \alpha_{j} \cdot 2^{k}}$ and $\widetilde{G}_{j}=g_{j}^{\widetilde{r}} / h^{b \cdot \widetilde{\alpha_{j}} \cdot 2^{k}}$; so, depending on $B$ 's challenge, encryptions of $s_{3}$ are prepared as in Game 2 or Game 3. Similar arguments work for $j^{*}=1,2,4$, and (7) follows. (The $\mathbf{O}\left(2^{-k}\right)$ term in (7) accounts for the statistical defect caused by choosing $\mathbb{G}_{\text {rnd }}$-exponents from $\mathbb{Z}_{N / 4}$, resp. $\mathbb{Z}_{N^{2} / 4}$.)

Using the definition of $u$ and $v$, our change in Game 3 implies $\widetilde{Z}=\left(u^{v k} v\right)^{r}$. $u^{\widetilde{r}} \cdot h^{K}$ when a key part $s_{j}$ is to be encrypted. (However, note that we still have $Z=\left(u^{v k} v\right)^{r \cdot N^{2}}$ in any case.) This means that $A$ still obtains information about the $s_{j}$ (beyond what is public from $p k$ ) from its KDM queries, but this information is limited to values $\operatorname{LAF}_{F p k, t}\left(\left[s_{j}\right]_{\mathbb{Z}_{p}^{\mathfrak{n}}}\right)$. We will now further cap this leaked information by making $\operatorname{LAF}_{F p k, t}(\cdot)$ lossy. Namely, in Game 4, we use the LAF trapdoor $F t d$ initially sampled along with $F p k$. Concretely, when preparing a ciphertext $C$ for $A$, we sample $t_{\mathrm{c}}$ using $t_{\mathrm{c}} \leftarrow \mathrm{FTag}\left(F t d, t_{\mathrm{a}}\right)$ for the corresponding auxiliary tag $t_{\mathrm{a}}=v k$. A simple reduction shows

$$
\operatorname{Pr}\left[\text { out }_{4}=1\right]-\operatorname{Pr}\left[\text { out }_{3}=1\right]=\operatorname{Adv}_{\mathrm{LAF}, C_{2}}^{\text {ind }}(k)
$$

for a suitable adversary $C_{2}$ on LAF's indistinguishability.

In Game 5, we reject all decryption queries of $A$ that re-use a verification key $v k$ from one of the KDM ciphertexts. To show that this change does not significantly affect $A$ 's view, assume a decryption query $C$ that re-uses a key $v k=v k^{*}$ from a KDM ciphertext $C^{*}$. Recall that $C$ contains a signature $\sigma$ of $X:=\left(\left(G_{j}, \widetilde{G}_{j}\right)_{j=1}^{2}, Z, \widetilde{Z}, C_{\mathrm{E}}, t_{\mathrm{c}}\right)$ under an honestly generated Sig-verification-key $v k=t_{\mathrm{a}}=t_{\mathrm{a}}^{*}=v k^{*}$. Since we assumed $t=\left(t_{\mathrm{c}}, t_{\mathrm{a}}\right)=\left(t_{\mathrm{c}}^{*}, t_{\mathrm{a}}^{*}\right)=t^{*}$, and $A$ is not allowed to query unchanged challenge ciphertexts for decryption, we must have $(X, \sigma) \neq\left(X^{*}, \sigma^{*}\right)$ for the corresponding message $X^{*}$ and signature $\sigma^{*}$ from $C^{*}$. Hence, Game 4 and Game 5 only differ when $A$ manages to forge a signature. A straightforward reduction to the strong OT-EUF-CMA security of Sig yields

$$
\operatorname{Pr}\left[\text { out }_{5}=1\right]-\operatorname{Pr}\left[\text { out }_{4}=1\right]=q(k) \cdot \operatorname{Adv}_{\mathrm{LAF}, F}^{\text {seuf-cma }}(k)
$$

for a forger $F$ against Sig that makes at most one signature query.

In Game 6.i (for $0 \leq i \leq q$ ), the first $i$ challenge ciphertexts are prepared using $Z=\widehat{g}^{N^{2}}$ and $\widetilde{Z}=\widehat{g} \cdot u^{\widetilde{r}} \cdot h^{K}$ (if a key component $s_{j}$ is to be encrypted), resp. $\widetilde{Z}=\widehat{g} \cdot u^{\widetilde{r}} \cdot h^{K+2^{k}[M]_{\mathbb{Z}}}$ (if a constant $M \in \mathcal{M}$ is to be encrypted) for an independently uniform $\widehat{g} \leftarrow \mathbb{G}_{\text {rnd }}$ drawn freshly for each ciphertext. Obviously, Game 6.0 is identical to Game 5: $\operatorname{Pr}\left[\right.$ out $\left._{6.0}=1\right]=\operatorname{Pr}\left[\right.$ out $\left._{5}=1\right]$. We will move 
from Game 6.i to Game 6. $(i+1)$ in several steps. During these steps, we denote with $C=\left(\left(G_{j}, \widetilde{G}_{j}\right)_{j=1}^{2}, Z, \widetilde{Z}, C_{\mathrm{E}}, t_{\mathrm{c}}, v k, \sigma\right)$ the $(i+1)$-st KDM ciphertext.

In Game 6.i.1, we change the $\mathbb{G}_{\text {rnd }}$ parts of $G_{1}, G_{2}$ from a Diffie-Hellman tuple (with respect to $g_{1}, g_{2}$ ) to a random tuple. Concretely, if an $s_{j}$ is to be encrypted, we set $\left(G_{1}, G_{2}\right)=\left(g_{1}^{r_{1}} / h^{\alpha_{1} \cdot 2^{k}}, g_{2}^{r_{2}} / h^{\alpha_{2} \cdot 2^{k}}\right)$; if a constant $M$ is encrypted, we set $\left(C_{1}, C_{2}\right)=\left(g_{1}^{r_{1}}, g_{2}^{r_{2}}\right)$, in both cases for independently uniform $r_{1}, r_{2} \leftarrow \mathbb{Z}_{N / 4}$. The $\mathbb{G}_{\mathrm{msg}}$ parts of $G_{1}, G_{2}$ are thus unchanged compared to Game 6.i. Note that the $\widetilde{G}_{j}$ are still prepared as $\widetilde{G}_{j}=g_{j}^{\widetilde{r}} / h^{\widetilde{\alpha} \cdot 2^{k}}$, resp. $\widetilde{G}_{j}=g_{j}^{\widetilde{r}}$. A straightforward reduction to the DDH assumption in $\mathbb{G}_{\text {rnd }}$ yields

$$
\sum_{i=1}^{q(k)}\left(\operatorname{Pr}\left[\text { out }_{6 . i}=1\right]-\operatorname{Pr}\left[\text { out }_{6 . i .1}=1\right]\right)=q(k) \cdot \operatorname{Adv}_{\mathbb{G}_{\text {rnd }}, D_{1}}^{\mathrm{ddh}}(k)+\mathbf{O}\left(2^{-k}\right)
$$

for a suitable $D_{1}$. The $\mathbf{O}\left(2^{-k}\right)$ error term accounts for the statistical difference caused by the choice of exponents $r_{j} \leftarrow \mathbb{Z}_{N / 4}$, which induces an only almostuniform distribution on group elements $g^{r_{j}}$. Note that at this point, $Z$ and $\widetilde{Z}$ are still computed as in (6), even if an $s_{j}$ is to be encrypted.

In Game 6.i.2, we compute $Z$ and $\widetilde{Z}$ as $Z=\widehat{g}^{N^{2}}$ and $\widetilde{Z}=\widehat{g} \cdot u^{\widetilde{r}} \cdot h^{K}$, resp. $\widetilde{Z}=$ $\widehat{g} \cdot u^{\widetilde{r}} \cdot h^{K+2^{k}[M]_{\mathbb{Z}}}$ for a fresh $\widehat{g} \leftarrow \mathbb{G}_{\text {rnd }}$. Thus, the difference to Game 6.i.1 is that we substitute a $\mathbb{G}_{\mathrm{rnd}}$-element computed as $G_{1}^{\left[s_{1}\right]_{\mathbb{Z}} \cdot v k+\left[s_{3}\right]_{\mathbb{Z}}} G_{2}^{\left[s_{2}\right]_{\mathbb{Z}} \cdot v k+\left[s_{4}\right]_{\mathbb{Z}}}$ with a fresh random $\widehat{g}$. To show that this change affects $A$ 's view only negligibly, it suffices to show that $A$ 's statistical information about

$$
\begin{aligned}
X:=\operatorname{dlog}_{g} & \left(G_{1}^{\left[s_{1}\right]_{\mathbb{Z}} \cdot v k+\left[s_{3}\right]_{\mathbb{Z}}} G_{2}^{\left[s_{2}\right]_{\mathbb{Z}} \cdot v k+\left[s_{4}\right]_{\mathbb{Z}}}\right) \\
& =\gamma_{1} r_{1}\left(\left[s_{1}\right]_{\mathbb{Z}} \cdot v k+\left[s_{3}\right]_{\mathbb{Z}}\right)+\gamma_{2} r_{2}\left(\left[s_{2}\right]_{\mathbb{Z}} \cdot v k+\left[s_{4}\right]_{\mathbb{Z}}\right) \bmod \left|\mathbb{G}_{\mathrm{rnd}}\right|
\end{aligned}
$$

(for some arbitrary generator $g$ of $\mathbb{G}_{\mathrm{rnd}}$ and $\gamma_{j}=\operatorname{dlog}_{g}\left(g_{j}\right)$ ) is negligible. This part will be rather delicate, since we will have to argue that both $A$ 's KDM queries and $A$ 's decryption queries yield (almost) no information about $X$.

First, observe that $A$ gets the following information about the $s_{j}$ :

- $p k$ reveals (through $u$ and $v$ ) precisely the two linear equations $\gamma_{1}\left[s_{1}\right]_{\mathbb{Z}}+$ $\gamma_{2}\left[s_{2}\right]_{\mathbb{Z}} \bmod \left|\mathbb{G}_{\text {rnd }}\right|$ and $\gamma_{1}\left[s_{3}\right]_{\mathbb{Z}}+\gamma_{2}\left[s_{4}\right]_{\mathbb{Z}} \bmod \left|\mathbb{G}_{\text {rnd }}\right|$ about the $s_{j}$, where the $\gamma_{j}$ are as above. For $r_{1} \neq r_{2}$, these equations are linearly independent of the equation that defines $X$. Hence, for uniform $r_{1}, r_{2}, X$ is (almost) independent of $p k$.

- By LAF's lossiness, KDM queries yield (through $C_{\mathrm{E}}=\mathrm{E}\left(K, \operatorname{LAF}_{F p k, t}\left(\left[s_{j}\right]_{\mathbb{Z}_{p}^{\mathfrak{n}}}\right)\right)$ in total at most one equation $\omega_{1} a_{j}+\omega_{2} b_{j}+\sum_{i=0}^{\mathfrak{n}-2} \omega_{3+i} c_{j, i} \bmod p$ for each $j$, where $\left(a_{j}, b_{j}, c_{j, 0}, \ldots, c_{j, \mathfrak{n}-3}\right):=\left[s_{j}\right]_{\mathbb{Z}_{p}^{\mathfrak{n}}}$, and the $\omega_{i}$ are the (fixed) coefficients from LAF's lossiness property. (Recall the encodings $\left[s_{j}\right]_{\mathbb{Z}},\left[s_{j}\right]_{\mathbb{Z}_{p}^{\mathfrak{n}}}$ of the $s_{j}=\left(a_{j}, b_{j}, c_{j}\right)$ from (5).) Hence, the $b_{j} \in \mathbb{Z}_{p \cdot 2^{k}}$ fully blind the information released about the $c_{j} \in \mathbb{Z}_{2^{k-2} N}$ through the KDM ciphertexts. Thus, KDM ciphertexts reveal no information about $c_{j} \bmod \left|\mathbb{G}_{\text {rnd }}\right|$ and hence also about $\left[s_{j}\right]_{\mathbb{Z}} \bmod \left|\mathbb{G}_{\mathrm{rnd}}\right|$.

Consequently, even given $p k$ and the KDM ciphertexts, $X$ is statistically close to independently uniform. This already shows that our change from Game 6.i.2 
affects $A$ 's view only negligibly if $A$ makes no decryption queries. It remains to show that decryption queries yield no additional information about the $s_{j}$.

To do so, let us say that a ciphertext $C$ is consistent iff there exist $r, \widetilde{r}$ with $\left(G_{j}, \widetilde{G}_{j}\right)=\left(g_{j}^{r}, g_{j}^{\widetilde{r}}\right)$ for both $j \in\{1,2\}$. Note that the decryption of a consistent ciphertext yields no information about the $s_{j}$ beyond $p k$. ( $p k$ and $r, \widetilde{r}$ determine the values $Z, Z^{\prime}$ computed during decryption; everything else follows from $Z^{\prime}$ and $C$.) So it suffices to prove the following lemma (which we do in [21]):

Lemma 3. In the situation of Game 6.i.l (for $\ell \in\{1,2\}$ ), let bad $_{\text {query.i. } \ell}$ be the event that $A$ places an inconsistent decryption query that is not rejected. Then

$$
\sum_{i=1}^{q(k)}\left(\operatorname{Pr}\left[\operatorname{bad}_{\text {query.i.1 }}\right]+\operatorname{Pr}\left[\operatorname{bad}_{\text {query.i.2 }}\right]\right) \leq 2 \cdot q(k) \cdot \operatorname{Adv}_{\mathrm{LAF}, F}^{\text {eva }}(k)+\mathbf{O}\left(2^{-3 k}\right) .
$$

for a suitable evasiveness adversary $F$ on LAF.

By our discussion above and Lemma 3, we obtain that

$$
\sum_{i=1}^{q(k)} \mid \operatorname{Pr}\left[\text { out }_{6 . i .2}=1\right]-\operatorname{Pr}\left[\text { out }_{6 . i .1}=1\right] \mid \leq 2 \cdot q(k) \cdot \operatorname{Adv}_{\mathrm{LAF}, F}^{\text {eva }}(k)+\mathbf{O}\left(2^{-3 k}\right)
$$

In Game 6.i.3, we reverse the change from Game 6.i.1. Concretely, we prepare the $G_{j}$ as $G_{j}=g_{j}^{r} / h^{\alpha_{j} \cdot 2^{k}}$, resp. $G_{j}=g_{j}^{r}$ for a single $r \leftarrow \mathbb{Z}_{N / 4}$. Another straightforward reduction to the DDH assumption in $\mathbb{G}_{\text {rnd }}$ yields that

$$
\sum_{i=1}^{q(k)}\left(\operatorname{Pr}\left[\text { out }_{6 . i .3}=1\right]-\operatorname{Pr}\left[\text { out }_{6 . i .2}=1\right]\right)=q(k) \cdot \operatorname{Adv}_{\mathbb{G}_{\mathrm{rnd}}, D_{2}}^{\mathrm{ddh}}(k)+\mathbf{O}\left(2^{-k}\right)
$$

for a suitable $D_{2}$. To close the hybrid argument, note that Games 6.i.3 and 6. $(\mathrm{i}+1)$ are identical.

In Game 7, we clear the $\mathbb{G}_{\mathrm{msg}}$-component of $\widetilde{Z}$ in all ciphertexts prepared for $A$. That is, instead of computing $\widetilde{Z}=\widehat{g} \cdot u^{\widetilde{r}} \cdot h^{K}$, resp. $\widetilde{Z}=\widehat{g} \cdot u^{\widetilde{r}} \cdot h^{K+[M]_{\mathbb{Z}}}$ for a freshly uniform $\widehat{g} \leftarrow \mathbb{G}_{\text {rnd }}$, we set $\widetilde{Z}=\widehat{g} \cdot u^{\widetilde{r}}$. (We stress that we still compute $Z=\widehat{g}^{N^{2}}$.) Since all $\widetilde{Z}$ already have an independently uniform $\mathbb{G}_{\text {rnd }}$-component, a straightforward reduction to the DCR assumption yields

$$
\operatorname{Pr}\left[\text { out }_{6 . q}=1\right]-\operatorname{Pr}\left[\text { out }_{7}=1\right]=\operatorname{Adv}_{\mathbb{Z}_{N^{3}}^{*}, E}^{\mathrm{ddr}}(k)+\mathbf{O}\left(2^{-k}\right)
$$

for a DCR distinguisher $E$. Note that because of the re-randomizability of DCR, there is no factor of $q(k)$, even though we substitute many group elements at once. However, since the precise order of $\mathbb{G}_{\text {rnd }}$ is not known, this re-randomization costs us an error term of $\mathbf{O}\left(2^{-k}\right)$.

In Game 8, we substitute the symmetric ciphertexts $C_{\mathrm{E}}$ in all KDM ciphertexts by encryptions of random messages. By our change in Game 7, we do not use the symmetric keys $K$ used to produce $C_{\mathrm{E}}$ anywhere else. Thus, a reduction to the IND-CPA security of (E, D) gives

$$
\operatorname{Pr}\left[\text { out }_{7}=1\right]-\operatorname{Pr}\left[\text { out }_{8}=1\right]=q(k) \cdot \operatorname{Adv}_{(\mathrm{E}, \mathrm{D}), G}^{\text {ind-cpa }}(k)
$$


for an IND-CPA adversary $G$. Note that in Game 8, $A$ 's view is independent of the challenge bit $b$ initially selected by the KDM challenger. Hence, we have $\operatorname{Pr}\left[\right.$ out $\left._{8}=1\right]=1 / 2$. Taking things together yields the theorem.

Acknowledgements. I would like to thank Hoeteck Wee for pointing out to me the connection between hash proof systems and KDM security. His interpretation of the schemes of [10] and [11] was the starting point for this work. I am grateful to Tibor Jager for very useful feedback and discussions. I am indebted to Baodong Qin, who pointed out two flaws (along with very helpful suggestions on how to fix them). Finally, I am thankful to the Crypto 2012 referees who found an annoying cyclic dependency in the PKE construction, and to the Eurocrypt 2013 referees for helpful comments.

\section{References}

[1] Acar, T., Belenkiy, M., Bellare, M., Cash, D.: Cryptographic agility and its relation to circular encryption. In: Gilbert, H. (ed.) EUROCRYPT 2010. LNCS, vol. 6110, pp. 403-422. Springer, Heidelberg (2010)

[2] Adão, P., Bana, G., Herzog, J., Scedrov, A.: Soundness of formal encryption in the presence of key-cycles. In: De Capitani di Vimercati, S., Syverson, P.F., Gollmann, D. (eds.) ESORICS 2005. LNCS, vol. 3679, pp. 374-396. Springer, Heidelberg (2005)

[3] Applebaum, B.: Key-dependent message security: Generic amplification and completeness. In: Paterson, K.G. (ed.) EUROCRYPT 2011. LNCS, vol. 6632, pp. 527-546. Springer, Heidelberg (2011)

[4] Applebaum, B., Cash, D., Peikert, C., Sahai, A.: Fast cryptographic primitives and circular-secure encryption based on hard learning problems. In: Halevi, S. (ed.) CRYPTO 2009. LNCS, vol. 5677, pp. 595-618. Springer, Heidelberg (2009)

[5] Barak, B., Haitner, I., Hofheinz, D., Ishai, Y.: Bounded key-dependent message security. In: Gilbert, H. (ed.) EUROCRYPT 2010. LNCS, vol. 6110, pp. 423-444. Springer, Heidelberg (2010)

[6] Bellare, M., Hofheinz, D., Yilek, S.: Possibility and impossibility results for encryption and commitment secure under selective opening. In: Joux, A. (ed.) EUROCRYPT 2009. LNCS, vol. 5479, pp. 1-35. Springer, Heidelberg (2009)

[7] Black, J., Rogaway, P., Shrimpton, T.: Encryption-scheme security in the presence of key-dependent messages. In: Nyberg, K., Heys, H.M. (eds.) SAC 2002. LNCS, vol. 2595, pp. 62-75. Springer, Heidelberg (2003)

[8] Boneh, D., Shen, E., Waters, B.: Strongly unforgeable signatures based on computational Diffie-Hellman. In: Yung, M., Dodis, Y., Kiayias, A., Malkin, T. (eds.) PKC 2006. LNCS, vol. 3958, pp. 229-240. Springer, Heidelberg (2006)

[9] Boneh, D., Canetti, R., Halevi, S., Katz, J.: Chosen-ciphertext security from identity-based encryption. SIAM Journal on Computing 36(5), 1301-1328 (2007)

[10] Boneh, D., Halevi, S., Hamburg, M., Ostrovsky, R.: Circular-secure encryption from decision diffie-hellman. In: Wagner, D. (ed.) CRYPTO 2008. LNCS, vol. 5157, pp. 108-125. Springer, Heidelberg (2008)

[11] Brakerski, Z., Goldwasser, S.: Circular and leakage resilient public-key encryption under subgroup indistinguishability - (or: Quadratic residuosity strikes back). In: Rabin, T. (ed.) CRYPTO 2010. LNCS, vol. 6223, pp. 1-20. Springer, Heidelberg (2010) 
[12] Brakerski, Z., Goldwasser, S., Kalai, Y.T.: Black-box circular-secure encryption beyond affine functions. In: Ishai, Y. (ed.) TCC 2011. LNCS, vol. 6597, pp. 201218. Springer, Heidelberg (2011)

[13] Camenisch, J.L., Lysyanskaya, A.: An efficient system for non-transferable anonymous credentials with optional anonymity revocation. In: Pfitzmann, B. (ed.) EUROCRYPT 2001. LNCS, vol. 2045, pp. 93-118. Springer, Heidelberg (2001)

[14] Camenisch, J., Chandran, N., Shoup, V.: A public key encryption scheme secure against key dependent chosen plaintext and adaptive chosen ciphertext attacks. In: Joux, A. (ed.) EUROCRYPT 2009. LNCS, vol. 5479, pp. 351-368. Springer, Heidelberg (2009)

[15] Cash, D., Green, M., Hohenberger, S.: New definitions and separations for circular security. Cryptology ePrint Archive, Report 2010/144 (2010), http://eprint.iacr.org/

[16] Cramer, R., Shoup, V.: Universal hash proofs and a paradigm for adaptive chosen ciphertext secure public-key encryption. In: Knudsen, L.R. (ed.) EUROCRYPT 2002. LNCS, vol. 2332, pp. 45-64. Springer, Heidelberg (2002)

[17] Galindo, D., Herranz, J., Villar, J.: Identity-based encryption with master keydependent message security and leakage-resilience. In: Foresti, S., Yung, M., Martinelli, F. (eds.) ESORICS 2012. LNCS, vol. 7459, pp. 627-642. Springer, Heidelberg (2012)

[18] Groth, J., Sahai, A.: Efficient non-interactive proof systems for bilinear groups. In: Smart, N.P. (ed.) EUROCRYPT 2008. LNCS, vol. 4965, pp. 415-432. Springer, Heidelberg (2008)

[19] Haitner, I., Holenstein, T.: On the (im)Possibility of key dependent encryption. In: Reingold, O. (ed.) TCC 2009. LNCS, vol. 5444, pp. 202-219. Springer, Heidelberg (2009)

[20] Hofheinz, D.: All-but-many lossy trapdoor functions. In: Pointcheval, D., Johansson, T. (eds.) EUROCRYPT 2012. LNCS, vol. 7237, pp. 209-227. Springer, Heidelberg (2012)

[21] Hofheinz, D.: Circular chosen-ciphertext security with compact ciphertexts. Cryptology ePrint Archive, Report 2012/150 (2012), http://eprint.iacr.org/

[22] Hofheinz, D., Kiltz, E.: Secure hybrid encryption from weakened key encapsulation. In: Menezes, A. (ed.) CRYPTO 2007. LNCS, vol. 4622, pp. 553-571. Springer, Heidelberg (2007)

[23] Hofheinz, D., Unruh, D.: Towards key-dependent message security in the standard model. In: Smart, N.P. (ed.) EUROCRYPT 2008. LNCS, vol. 4965, pp. 108126. Springer, Heidelberg (2008)

[24] Kurosawa, K., Desmedt, Y.: A new paradigm of hybrid encryption scheme. In: Franklin, M. (ed.) CRYPTO 2004. LNCS, vol. 3152, pp. 426-442. Springer, Heidelberg (2004)

[25] Malkin, T., Teranishi, I., Yung, M.: Efficient circuit-size independent public key encryption with KDM security. In: Paterson, K.G. (ed.) EUROCRYPT 2011. LNCS, vol. 6632, pp. 507-526. Springer, Heidelberg (2011)

[26] Naor, M., Yung, M.: Public-key cryptosystems provably secure against chosen ciphertext attacks. In: 22nd ACM STOC. ACM Press (May 1990)

[27] Peikert, C., Waters, B.: Lossy trapdoor functions and their applications. In: Ladner, R.E., Dwork, C. (eds.) 40th ACM STOC, pp. 187-196. ACM Press (May 2008)

[28] Waters, B.: Efficient identity-based encryption without random oracles. In: Cramer, R. (ed.) EUROCRYPT 2005. LNCS, vol. 3494, pp. 114-127. Springer, Heidelberg (2005) 